\title{
Infecção urinária na gestação e as repercussões ao recém-nascido: uma revisão integrativa
}

\author{
Urinary tract infection in pregnancy and its repercussions for the newborn: an integrative \\ review
}
Infección del tracto urinario en el embarazo y su repercusión en el recién nacido: una revisión integradora

Letícia Pereira de Oliveira ${ }^{1}$, Raiele Maria Alves de Araujo ${ }^{1}$, Mariana Delfino Rodrigues ${ }^{1 *}$.

\section{RESUMO}

Objetivo: Discutir e revisar sobre a infecção urinária na gestação e as repercussões ao recém-nascido. Métodos: Trata-se de uma revisão integrativa de literatura composta por oito artigos e uma tese disponíveis nas bases de dados da Biblioteca Virtual em Saúde Brasil (BVS) e Literatura Latino-Americana e do Caribe em Ciências da Saúde (LILACS), sendo selecionados após a definição dos descritores "Gravidez AND trato urinário". Resultados: Da análise do conteúdo das publicações, emergiram 3 categorias temáticas, sendo: a) Condições socioeconômicas; b) Faixa etária das gestantes; c) Complicações da infecção urinária ao recémnascido. Foi possível verificar que gestantes de baixa renda têm mais chance de desenvolverem ITU, a faixa etária de maior acometimento é entre 18 e 29 anos. A prematuridade foi a repercussão ao recém-nascido apontada mais frequentemente quando relacionada à ITU. Vale ressaltar que, $89 \%$ dos artigos revisados afirmam que há a necessidade de melhora do manejo da gestante durante o pré-natal, pois os eventos estudados podem ser evitados através do rastreamento recomendado pelo Ministério da Saúde. Considerações finais: Entre as publicações revisadas foi possível analisar que a principal complicação ao recém-nascido relacionada à ITU gestacional é a prematuridade.

Palavras-chave: Trato urinário, Gravidez, Pré-natal, Recém-nascido.

\section{ABSTRACT}

Objective: Discuss and review urinary tract infection during pregnancy and its repercussions for the newborn. Methods: This is an integrative literature review composed of eight articles and a thesis available in the databases of the Virtual Health Library (VHL) and Latin American and Caribbean Health Sciences Literature (LILACS), being selected after the definition of the descriptors "Gravidez AND trato urinário" and used the inclusion and exclusion criteria of the research. Results: From the analysis of the content of the publications, three thematic categories emerged, as follows: a) Socioeconomic conditions; b) Age group of pregnant women; c) Complications of urinary infection to the newborn. It was possible to verify that low-income pregnant women are more likely to develop UTI, the age group most affected is between 18 and 29 years. Prematurity was the repercussion to the newborn most frequently indicated when related to UTI. It is worth mentioning that $89 \%$ of the reviewed articles state that there is a need to improve the management of pregnant women during prenatal care, because the events studied can be avoided through screening recommended by the Ministry of Health. Final considerations: The repercussions for the newborn are low weight and prematurity. It is verified the need for other studies addressing the relationship of gestational UTI with complications to newborns that evidences the present relationship and can strengthen the approach of importance in the gestational period.

Key words: Urinary tract, Pregnancy, Prenatal, Newborn.

\footnotetext{
${ }^{1}$ Centro Universitário Aparício Carvalho (FIMCA), Porto Velho - RO. *E-mail: mdr.enf@gmail.com
} 


\section{RESUMEN}

Objetivo: Discutir y revisar la infección del tracto urinario durante el embarazo y sus repercusiones en el recién nacido. Métodos: Es una revisión integradora de la literatura compuesta por ocho artículos y una tesis disponible en las bases de datos de la Biblioteca Virtual en Salud Brasil (BVS) y Literatura Latinoamericana y del Caribe en Ciencias de la Salud (LILACS), siendo seleccionada luego de definir los descriptores "Embarazo y tracto urinario". Resultados: Del análisis del contenido de las publicaciones surgieron 3 categorías temáticas, como sigue: a) Condiciones socioeconómicas; b) Grupo de edad de las mujeres embarazadas; c) Complicaciones de la infección del tracto urinario al recién nacido. Se pudo comprobar que las mujeres embarazadas de bajos ingresos tienen más probabilidades de desarrollar ITU, el grupo de edad más afectado es entre 18 y 29 años. La prematuridad fue la repercusión en el recién nacido más señalada en relación con la ITU. Cabe mencionar que el $89 \%$ de los artículos revisados señalan que existe la necesidad de mejorar el manejo de la gestante durante la atención prenatal, ya que los eventos estudiados pueden evitarse mediante el cribado recomendado por el Ministerio de Salud. Consideraciones finales: Entre los revisados publicaciones se pudo analizar que la principal complicación del recién nacido relacionada con la IU gestacional es la prematuridad.

Palabras clave: Tracto urinario, Embarazo, Prenatal, Recién nacido.

\section{INTRODUÇÃO}

A Infecção do Trato Urinária (ITU) denomina-se como a invasão e multiplicação de uropatógenos que causam alterações fisiológicas e podem gerar uma tríade sintomática apresentada por polaciúria, disúria e retenção urinária, ela pode ser classificada como cistite ou uretrite (infecção baixa), pielonefrite (infecção alta) ou bacteriúria assintomática (quando é identificado a presença de bactérias na urina acima de $100.000 \mathrm{col} / \mathrm{ml}$ sem qualquer sintoma urinário) (CANÇADO MAP, et al., 2018).

A ITU é uma das infecções mais recorrentes na população brasileira, dados epidemiológicos apontam que as mulheres têm mais chances de terem infecção urinária comparada aos homens, estando mais suscetíveis durante o período gestacional pois as alterações fisiológicas, anatômica e hormonais, como: hipertrofia, hipotonicidade e hipomotilidade da musculatura uretral, modificação da posição da bexiga durante o segundo trimestre, aumento do débito urinário, estase urinário, refluxo vesicouretral e alteração do $\mathrm{pH}$ da urina (tornando-se mais alcalina) contribuem para a proliferação de bactérias que ocasionam a ITU (ALVES DMS, et al., 2016; OLIVEIRA RA, et al., 2016; RIBEIRO RM 2001; VARELA PLR, et al., 2015).

A ocorrência desta infecção em gestantes pode causar complicações graves e gerar vários transtornos durante a gestação, assim como no período perinatal quando não diagnosticada e tratada corretamente, pois pode atingir o recém-nascido (RN), assim para evitar tais desarranjos é de suma importância a realização de um pré-natal de qualidade (MATA KS, et al., 2014).

O Ministério da Saúde (2012) indica a solicitação do exame de urina tipo I e urocultura entre os exames de rotina do pré-natal na primeira consulta, e no primeiro, segundo e terceiro trimestre para detectar a infecção urinária e trata-la precocemente, visto a presença de ITU assintomática, sendo possível o diagnóstico através de exames laboratoriais.

O rastreio descrito acima é de suma importância, visto que a ITU sendo um fator de risco gestacional, podendo resultar em: a mortalidade materna, pielonefrite, choque séptico, pré-eclâmpsia e restrição ido crescimento intrauterino. No recém-nascido ela está diretamente ligada com o parto prematuro, ruptura prematura da membrana e baixo peso ao nascer. Considerando que tais fatores estão relacionados à taxa de mortalidade infantil, através de um manejo adequado é possível minimizar as causas dos óbitos infantis relacionado à ITU materna (SANTOS JN, et al., 2017; LAI LJ, et.al., 2017; LISBOA L, et al., 2015).

Estudos indicam que a ITU e as complicações neonatais atingem, principalmente, gestantes de baixa renda, que não terminaram o ensino fundamental, pois elas têm menos acesso aos serviços de saúde, 
condições de moradia precária e tais fatores também estão relacionados a prática de higiene inadequada (MATA KS, et al., 2014). Diante de tais fatos, este estudo visou analisar sobre a infecção urinária na gestação e as repercussões ao recém-nascido.

\section{MÉTODOS}

Para alcançar o objetivo do estudo utilizou-se o método de revisão integrativa seguindo os passos que a mesma compõe, sendo: identificação do problema; coleta de dados; avaliação de dados; análise e interpretação; interpretação dos resultados; síntese do conhecimento ou também chamado apresentação da revisão (SOUZA MT, et al., 2010).

Com o propósito de atender a primeira fase da pesquisa, formulou-se a pergunta norteadora: "Quais as repercussões ao recém-nascido ocasionadas pela infecção urinária materna durante o período gestacional?"

A coleta de dados ocorreu no mês Agosto do ano de 2020 nas bases de dados da Biblioteca Virtual em Saúde Brasil (BVS) e na Literatura Latino-Americana e do Caribe em Ciências da Saúde (LILACS), onde se utilizou como base os descritores em Ciências da Saúde (DeCS), sendo empregado das seguinte forma: "Gravidez AND trato urinário". Desta forma foram encontrados 1286 publicações científicas na BVS Brasil e 160 publicações na LILACS.

Os critérios de inclusão foram publicações em português disponíveis nas bases de forma completa e nos últimos 10 anos, após a filtragem permaneceram 32 publicações na BVS e 28 na LILACS e os critérios de exclusão foram estudos duplicados e os artigos que não contemplavam o objeto de estudo como demonstrado na Figura 1.

Figura 1 - Fluxograma de pesquisa de estudos.

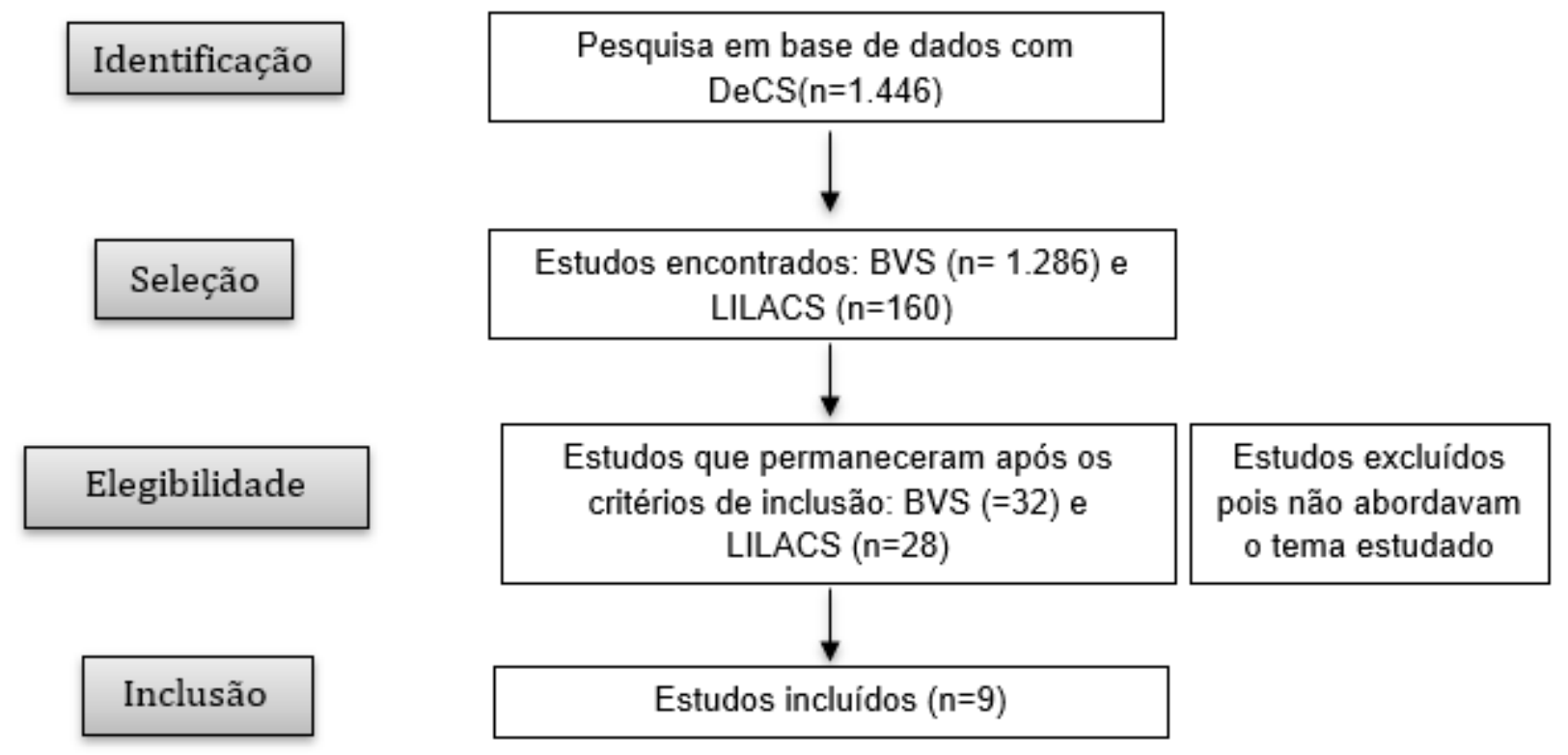

Fonte: Oliveira LP, et al., 2020.

$\mathrm{Na}$ avaliação dos dados, que sucedeu após a leitura exploratória dos resumos dos artigos, foram selecionadas 9 publicações encontradas em ambas as plataformas e possuíam afinidade com 0 tema proposto.

A quarta fase foi a análise e interpretação, que se formou no arranjo dos resultados dos estudos selecionados, intercalado com discussão teórica. A quinta fase abrangeu a interpretação dos resultados a partir da elaboração de tabelas com dados relevantes das pesquisas e por fim, na sexta fase, sucede à síntese 
do conhecimento, ou também a apresentação da revisão, compondo as etapas realizadas e os resultados corroborados com a análise dos artigos incluídos.

\section{RESULTADOS E DISCUSSÃO}

Foram analisados 9 estudos conforme os critérios de inclusão mencionados anteriormente, quanto ao período de publicação, dois artigos foram publicados em 2015 e dois em 2018, entre 2011 e 2014 e no ano de 2016 foram publicados um artigo em cada ano, sendo que 33\% apresentam Qualis B3, conforme demonstra o Quadro 1.

Quadro 1 - Distribuição das referências bibliográficas obtidas nas bases de dados.

\begin{tabular}{|c|c|c|}
\hline $\mathbf{N}$ & Autores (Ano) & $\begin{array}{l}\text { Principais achados } \\
\end{array}$ \\
\hline 1 & MENEZZI AMED, et al. (2016) & $\begin{array}{l}\text { Dos resultados verificou } 69 \% \text { de óbitos sem fatores de risco } \\
\text { associados e mais de } 11 \% \text { relacionados a múltiplas } \\
\text { intercorrências. }\end{array}$ \\
\hline 2 & OLIVEIRA CS, et al. (2015) & $\begin{array}{l}\text { Longos períodos de internação após o nascimento estão } \\
\text { intimamente relacionados com baixo peso e idade } \\
\text { gestacional ao nascimento.. }\end{array}$ \\
\hline 3 & MATA KL, et al. (2014) & $\begin{array}{l}\text { Conclui-se que o trabalho de parto prematuro foi a principal } \\
\text { complicação causada pela infecção do trato urinário. }\end{array}$ \\
\hline 4 & BUENDGENS BB (2015) & $\begin{array}{l}\text { Conclui-se que a taxa de nascimento de prematuros tardios foi } \\
\text { equivalentes à populações de estudos internacionais. }\end{array}$ \\
\hline 5 & FALAVINA LP, et al. (2018) & $\begin{array}{l}\text { O estudo identificou elevada prevalência de hospitalização na } \\
\text { gestação, especialmente em gestantes com parto financiado } \\
\text { pelo setor público de atenção à saúde. }\end{array}$ \\
\hline 6 & SANTOS IDL, et al. (2018) & $\begin{array}{l}\text { Acredita-se que as tecnologias em saúde podem ser utilizadas } \\
\text { para a estruturação da assistência para a prevenção do near } \\
\text { miss materno. }\end{array}$ \\
\hline 7 & $\begin{array}{l}\text { ACKENHAAR AA e ALBERNAZ } \\
\text { P (2013) }\end{array}$ & $\begin{array}{l}\text { A alta taxa de internação hospitalar reflete a falta de efetividade } \\
\text { no rastreamento da infecção urinária durante a gestação. }\end{array}$ \\
\hline 8 & MACHADO JR et al. (2012) & $\begin{array}{l}\text { Os diferentes padrões de corioamnionite estão relacionados a } \\
\text { diferentes manifestações clínicas materno-fetais, influenciando } \\
\text { nas condições de vida do recém-nascido e na gravidade de } \\
\text { lesões morfológicas encontradas em natimortos. }\end{array}$ \\
\hline 9 & BAUMGARTEN MCS, et al. (2011) & $\begin{array}{l}\text { As ITUs no período gestacional são comuns pelas alterações } \\
\text { anatômicas, as alterações hormonais e a mudança do pH } \\
\text { facilitando a entrada de micro-organismos no aparelho } \\
\text { geniturinário feminino, sendo de grande importância o } \\
\text { acompanhamento pré-natal e os exames clínicos regulares. }\end{array}$ \\
\hline
\end{tabular}

Fonte: Oliveira LP, et al., 2020.

Da análise do conteúdo das publicações, emergiram 3 categorias temáticas, sendo: a) Condições socioeconômicas; b) Faixa etária das gestantes; c) Complicações da infecção urinária ao recém-nascido. Na categoria "Condições socioeconômicas", os seguintes autores desenvolveram estudos que apresentam as ideias em comum mencionadas abaixo:

O estudo de Menezzi AMED, et al. (2016) traz as principais causas sobre o Óbito Fetal (OF), possíveis de complicações da Infecção no Trato Urinário (ITU). A pesquisa revela que quando identificada o OF relacionada a ITU, observa-se a frequência de fatores associados a países em desenvolvimento, ou seja, em países de baixa e média renda. Além disso pode estar relacionado à pobreza, falta de educação, idade materna e falta de cuidados.

Oliveira CS, et al. (2015), fazem relação da prematuridade às condições socioeconômicas desfavoráveis, além das causas gestacionais, os autores afirmam que as informações errôneas sobre os cuidados durante a gestação, as condições precárias de assistência à saúde e o acompanhamento de pré-natal insatisfatório, são prejudiciais ao desenvolvimento embrionário. 
Falavina LP, et al. (2018), alegam que gestantes adolescentes ou idade avançada, de classes sociais desfavoráveis e com baixa escolaridade são mais propensas a desenvolverem complicações na gestação. Hackenhaar AA e Albernaz EP (2013), revelam que a bacteriúria assintomática acomete em maiores taxas nas gestantes de menor nível socioeconômico, não alfabetizadas, multíparas e mais jovens.

Segundo Baumgarten MCS, et al. (2011), os fatores de risco para bacteriúria assintomática são comportamento sexual, aumento da idade, multiparidade, susceptibilidade individual, baixo nível socioeconômico e história de ITU's na infância. O estudo de Mata KS, et al. (2014), contribui para identificar que as condições socioeconômicas são possíveis determinantes para o desenvolvimento de ITU, onde verificou as seguintes características: gestantes que tem o ensino fundamental incompleto $(41,25 \%)$, como ocupação principal foi declarado o serviço doméstico (51,25\%), com renda familiar de 1 a 3 salários mínimos $(33,75 \%)$.

A pesquisa de Falavina LP, et al. (2018), trouxe alguns dados referentes às variáveis sociodemográficas: as gestantes com parto financiado pelo SUS apresentaram características menos favoráveis, como maior proporção de gestantes adolescentes ( $18,1 \%$ SUS e $5,5 \%$ não SUS), gestantes com baixa renda ( $39,3 \%$ SUS e $7,1 \%$ não SUS), com menor escolaridade ( $91,8 \%$ SUS e $49,2 \%$ não SUS), raça/cor preta e parda (63,2\% SUS e $31,3 \%$ não SUS) e com menos de sete consultas de pré-natal (25,3\% SUS e 7,8\% não SUS).

Nos estudos que apresentaram os dados sociodemográficos é possível dizer que as informações apontam que gestantes de baixa renda têm mais chance de serem acometidas pela ITU durante a gravidez. Alguns estudos ressaltaram que a maioria das gestantes de baixa renda e com menor escolaridade possuem hábitos de higiene inadequado devido à falta de informação, vivem em condições precárias e têm menos acesso aos serviços de saúde, o que reflete no menor número de consultas durante o pré-natal e a falta de exames laboratoriais, prejudicando o manejo adequado para detecção de intercorrências que estão diretamente ligadas as complicações aos recém-nascidos. (MATA KS, et al., 2014; OLIVEIRA CS, et al., 2015; HACKENHAAR AA e ALBERNAZ EP, 2013).

Em seu estudo Hackenhaar AA e Albernaz EP (2013) concluem que há a necessidade de melhorar o rastreamento da bacteriúria assintomática para as mulheres de baixa renda e com escolaridade baixa pois a internação hospitalar para o tratamento da ITU durante a gravidez é mais elevada entre este grupo.

Na categoria "Faixa Etária", Menezzi AMED, et al. (2016) afirmam que a faixa etária considerada fator de risco é de 18 a 29 anos, ou seja, 35\% das gestantes encontravam-se nas idades de risco. Por infecção do trato urinário 3,8\% tiveram intercorrência durante a gravidez. Corroborando com o estudo de Mata KS, et al. (2014), na qual verificaram que a maioria se encontrava entre $20-29$ anos (48,75\%). Falaviana LP et al. (2018), revelaram que de 928 gestantes entrevistadas, 223 tinham a faixa etária entre 20-34 anos.

Quanto a idade, dois estudos apresentaram que a faixa etária de maior acometimento em ITU é entre 18 e 29 anos, apenas em um estudo foi apresentado maior acometimento em gestantes entre 20- 34 anos. Essa faixa etária vem de encontro ao ser a faixa etaria reprodutiva da mulher.

Sobre a categoria "Complicações", Menezzi AMED, et al. (2016), revelam que o óbito fetal é uma complicação decorrente da infecção urinária, da pesquisa 3,8\% tiveram ITU como intercorrência gestacional. $\mathrm{O}$ ocorrido pode trazer à gestante transtornos clínicos, obstétricos e emocionais. Na pesquisa não foram verificadas outras infecções maternas e intrauterinas além da ITU, ainda assim a mesma é tida como causa importante de morte fetal. O estudo de Menezzi AMED et al. (2016) também conclui que entre os óbitos fetais, $69 \%$ não tinham fatores de risco associados e $11 \%$ estavam relacionados a múltiplas intercorrências.

Para Buendgens BB (2015), o nascimento pré-termo é fator de risco ocasionado devido a infecção urinária. Os prematuros possuem características, entre estes estão distúrbios respiratórios provocados pelo desenvolvimento pulmonar incompleto, limitações na capacidade digestiva e podem ter dificuldades e problemas na alimentação, devido a coordenação sucção/deglutição/respiração imatura e baixo peso.

No estudo Buendgens BB (2015) de estudo observou que as doenças mais infeciosas mais frequentes foram do trato urinário e vaginal, de 288 mães de prematuro tardio, 77 tiveram intercorrências na gestação 
devido a ITU. A pesquisa de Oliveira CS, et al. (2015), também retrata a prematuridade, $28 \%$ das gestantes foram diagnosticadas com patologia referida.

No estudo de Mata KS, et al. (2014), em relação ao desenvolvimento de complicações pela gestante, revelou que a maioria $(57,5 \%)$ desenvolveu trabalho de parto prematuro, sendo este monitorado para que não evoluísse para o parto prematuro, seguida da pielonefrite $(13,75 \%)$ a segunda complicação mais encontrada.

Oliveira CS, et al. (2015), afirma que o cuidado a este tipo de RN torna-se necessário durante e após o parto, já que estes são muito vulneráveis a manipulação e ao desenvolvimento de doenças, o suporte hospitalar é importante, para reduzir a permanência no hospital. Falavina LP, et al. (2018), diz que a infecção urinária é um dos casos mais graves de hospitalização na gestação, teve como resultado, 76 gestantes tiveram como causa de internação, a infecção do trato urinário.

Destaca-se as palavras de Hackenhaar AA e Albernaz EP (2013), que 2,9\% internaram-se para o tratamento da ITU, este estudo encontrou associação entre internação hospitalar por ITU da gestante e a ocorrência da ameaça de trabalho de parto pré-termo, porém ao relacionar com o nascimento prematuro o autor ressalta que tal infecção não esteve associada com a intercorrência citada.

Em relação ao baixo peso, entre os RNs estudados por Buedgens BB (2015) metade deles apresentaram tal complicação ao nascer, porém não foi possível concluir se tal fato estava relacionado a ITU.

Entre os recém-nascidos pré-termos estudos por Oliveiras CS, et al. (2015) ressalta que a média do peso ao nascer foi de $1.478 \mathrm{~g}$ porém não foi perceptível observar se tal fato está relacionado a infecção urinária durante a gravidez, pois o dado exposto inclui RNs de gestantes que também não apresentaram ITU gestacional. Já em sua revisão, Baumgarten MCS, et al. (2011) ao contrapor o grupo que apresentou ITUs no período gestacional ao grupo de mulheres que não tiveram esta intercorrência, a maior taxa de prematuros com baixo peso ao nascer foi do primeiro grupo de gestantes.

Segundo Baumgarten MCS, et al. (2011), a cistite bacteriana está ligada ao parto prematuro, associada com a morbidade materna e fetal, a pielonefrite aguda é a forma mais grave de ITUs e a indicação mais comum de internação pré-parto. Os fatores de risco para o desenvolvimento de pielonefrite incluem os da BA e cistite, bem como uma história de pielonefrites, malformações do trato urinário e cálculos renais, 0 autor destaca que essas patologias no período gestacional são comuns pelas alterações anatômicas, as alterações hormonais e a mudança do $\mathrm{pH}$ promovendo a entrada de micro-organismos no aparelho geniturinário feminino.

O estudo de Machado JR, et al. (2012), apresenta a influência das intercorrências maternas e fetais nos grau de corioamnionite, entre elas está a bacteriúria, e está ligada a prematuridade. A mesma é uma inflamação placentária que interfere na interação materno-fetal. Observa-se que $12(15,0 \%)$ tiveram intercorrência materna por ITU, foram considerados nascidos a termo $65(72,2 \%)$ crianças e prematuras 25 $(27,8 \%)$, nos casos de infecção do trato urinário tiveram: grau I: $16,7 \%$; grau II: $58,3 \%$; grau III: 0,0\%; grau IV: $25,0 \%$ e grau V: $0,0 \%$.

Em relação as complicações maternas, alguns artigos destacaram que a a pielonefrite e infecção puerperal são agravos que afetam a mãe no pós-parto associadas com a ITU gestacional (MATA KS, et al., 2014; SANTOS IDL, et al., 2018; BUENDGENS BB, 2015). Assim dos estudos analisados é possível concluir que, $55 \%$ dos artigos apresentam a prematuridade como a principal complicação relacionada a ITU gestacional.

O estudo de Hackenhaar AA e Albernaz EP (2013) associou a internação ocasionada por ITU gestacional com o a ameaça de trabalho de parto pré-termo. Menezzi AMED, et al. (2016) e Baumgarten MCS et al. (2011) apresenta a infecção do trato urinário como um dos fatores de risco para a mortalidade fetal. Já Machado JR (2012) relata que a infecção do trato urinário no período gestacional é uma das intercorrências que aumenta o risco de corioamnionite.

Falavino LP, et al. (2018) e Oliveira CS, et al. (2015) associam a necessidade internação do RN com a ITU gestacional. Quanto ao baixo peso ao nascer apenas o estudo de Hackenhaar AA e Albernaz EP (2013) 
apresenta dados que associam a ITU à complicação referida. Vale ressaltar que $89 \%$ dos artigos revisados afirmam a necessidade de melhora do manejo da gestante durante o pré-natal, pois os eventos estudados podem ser evitados através do rastreamento recomendado pelo Ministério da Saúde.

\section{CONSIDERAÇÕES FINAIS}

Entre as publicações revisadas foi possível analisar que a principal complicação ao recém-nascido relacionada à ITU gestacional é a prematuridade, sendo a infecção do trato urinário é um fator de risco para a corioamnionite, baixo peso ao nascer, internação dos recém-nascidos e óbito fetal. As mulheres entre a faixa etária 18-34 anos, com baixa renda e pouca escolaridade são as que mais apresentam casos de ITU gestacional, consequentemente é a população materna que mais apresentam complicações nos RNs. Esperase que outras pesquisas abordando o tema estudado sejam realizadas, a fim de possibilitar a ampliação do conhecimento sobre as causas da relação entre as complicações aos recém-nascidos com a ITU gestacional para a melhoria da assistência durante o pré-natal.

\section{REFERÊNCIAS}

1. ALVES DMS, et al. Infecções comunitárias do trato urinário: prevalência e susceptibilidade aos antimicrobianos na cidade de Florianópolis. Revista Brasileira Medicina da Família e Comunidade, 2016; 11 (38): 1-12.

2. BAUMGARTEN MCS, et al. Infecção Urinária: Uma Revisão da Literatura. UNOPAR Cient Ciência Biologia Saúde, 2011; 13: 333-42.

3. BRASIL. Ministério da Saúde. Secretaria de Atenção à Saúde. Departamento de Atenção Básica. Atenção ao prénatal de baixo risco 1 ed. Brasília: Editora do Ministério da Saúde, 2012. Disponível em: http://bvsms.saude.gov.br/bvs/publicacoes/cadernos_atencao_basica_32_prenatal.pdf. Acessado em: 10 de agosto de 2020.

4. BUENDGENS BB. Fatores maternos na ocorrência da prematuridade tardia em um hospital universitário. Dissertação (Mestrado em Enfermagem) - Programa de Pós-graduação em Enfermagem. Escola de Enfermagem da Universidade Federal do Rio Grande do Sul, Rio Grande do Sul, Porto Alegre, 2015; 65p.

5. CANÇADO MAP, et al. Infecção do Trato Urinário. Atualização Terapêutica: Diagnóstico e Tratamento. 26 ed. São Paulo: Artes Médicas, 2018; 351- 354p.

6. FALAVINA LP, et al. Hospitalização durante a gravidez segundo financiamento do parto: um estudo de base populacional. Revista de Escola de Enfermagem da USP. 2018; 52: e 03317.

7. HACKENHAAR AA, ALBERNAZ EP. Prevalência e fatores associados à internação hospitalar para tratamento da infecção do trato urinário durante a gestação. Revista Brasileira de Ginecologia e Obstetricia. 2013; 35(5).

8. LAI YJ, et al. Asymptomatic pyuria in pregnant women during the first trimester is associated with an increased risk of adverse obstetrical outcomes. Taiwanese Journal of Obstetrics and Gunecology, 2017; 56: 192-195.

9. LISBOA L, et al. Mortalidade infantil: principais causas evitáveis na região Centro de Minas Gerais, $1999-2011$. Epidemiol. Serv. Saúde., 2015, 24 (4):711-720

10. MACHADO JR, et al. Influência das intercorrências maternas e fetais nos diferentes graus de corioamnionite. Revista Brasileira de Ginecologia e Obstetricia. 2012; 34(4).

11. MATA KS, et al. Complicações Causadas pela Infecção do Trato Urinário na Gestação. Revista espaço para a saúde. 2014; 15(4): 57-63.

12. MENEZZI AMED, et al. Vigilância do óbito fetal: estudo das principais causas. O Mundo da Saúde. 2016; 40(2): 20812.

13. OLIVEIRA CS, et al. Perfil de recém-nascidos pré-termo internados na unidade de terapia intensiva de hospital de alta complexidade. ABCS Health Sciences. 2015; 40(1): 28-32.

14. OLIVEIRA RA, et al. Perfil de suscetibilidade de uropatógenos em gestantes atendidas em um hospital no sudeste do Estado do Pará, Brasil. Revista Pan-Amaz Saúde. 2016; 7(3): 43-50.

15. RIBEIRO RM, et al. Uroginecologia e cirurgia vaginal. São Paulo: Roca, 2001; 279p.

16. SANTOS IDL, et al. Near Missmaterno no trabalho de parto e parto à luz das tecnologias em saúde. Revista Escola de Enfermagem USP, 2018; 52: e03409.

17. SANTOS JN, et al. Internacional Nursing Congress. Infecção do Trato Urinário na gravidez: Complicações e Intervenções de Enfermagem. Anais. Aracaju: Universidade Tiradentes, 2017.

18. SOUZA MT, et al. Revisão integrativa: o que é e como fazer. Eisntein. 2017; 8(1): 102-6.

19. VARELA PLR, et al. Intercorrências na gravidez em puérperas brasileiras atendidas nos sistemas público e privado de saúde. Revista Latino-Americana de Enfermagem. 2017; 25: 1-9. 